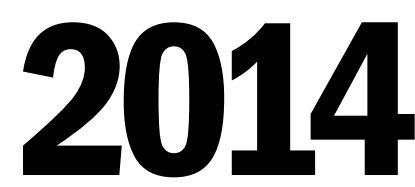

volume 11 | issue 1

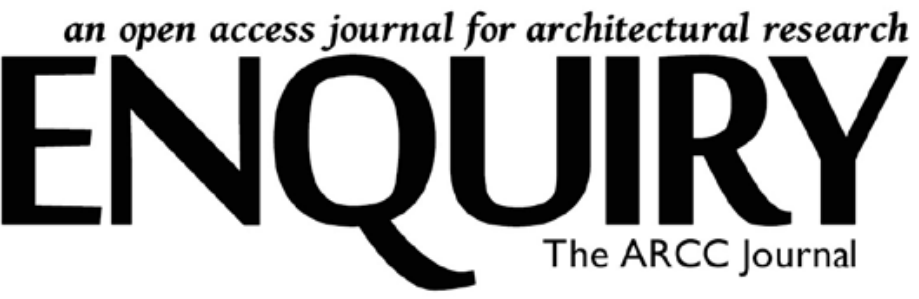

\title{
GRAPHIC ECOLOGIES
}

\author{
Brook Weld Muller
}

\begin{abstract}
This essay speculates about the influence of strategic approaches to graphic representation associated with critical environmental engagement and that build from the idea of works of architecture as contributors to ecologically functional cities. It investigates the utility of an approach where partial, built up and fragmented graphics describe inclusive, open-ended possibilities for making architecture that marry rich experience and responsive performance. Such an aphoristic approach to crafting drawings involves complex layering, conscious absence and the embracing of tension. The generation of imagery characterized by the notion of 'loose precision' invites collaborators and external audiences to adopt a more active and critical stance with regard to the environmental goals of a project that can, it is hypothesized, lead to more transformative and ecologically responsive architectures.
\end{abstract}

\section{INTRODUCTION}

In this essay I speak to the relationship between opportunities to aggressively engage ecological concerns in architecture and the priorities of sustainability-minded designers as evidenced by the drawings they produce. I look to innovative contemporary designers who construct drawings as critical mediums of expression of both environmental circumstances and suitable design response. How do environmental imperatives shape the

\section{Permissions and copyright}

Authors retain copyright and grant the journal right of first publication with the work simultaneously licensed under a Creative Commons Attribution License that allows others to share the work with an acknowledgement of the work's authorship and initial publication in this journal (Attribution-ShareAlike).

Creative Commons Attribution 3.0 Unported (CC BY 3.0)

You are free to: Share - copy and redistribute the material in any medium or format. Adapt — remix, transform, and build upon the material for any purpose, even commercially. The licensor cannot revoke these freedoms as long as you follow the license terms.

Under the following terms: Attribution - You must give appropriate credit, provide a link to the license, and indicate if changes were made. You may do so in any reasonable manner, but not in any way that suggests the licensor endorses you or your use.

No additional restrictions - You may not apply legal terms or technological measures that legally restrict others from doing anything the license permits.

How to cite:

Muller, Brook W. 2014. "Graphic Ecologies." Enquiry 11 (1): 33-39. types and nature of drawings green minded architects produce? In what ways do more 'fragmented' graphics, versus those of fuller resolution, provide indication of deep interest in the connectivity between individual, site-scale works of architecture and broader environmental systems? How do these artifacts of communication in the initial phases of a project impact understandings of what is possible and how do these insights cycle back into subsequent processes of design?

In this essay I endeavor to build a conceptual background and framework for a more comprehensive trajectory of inquiry that I wish to encourage and engage in (motivating for instance studiobased explorations and associated research in design pedagogy). I look at examples of drawings produced by firms considered on the vanguard of green architecture and offer a set of observations as to how these artifacts prompt new ways of thinking about design relative to imperatives of sustainability. This undertaking builds from recognition that medium influences design thinking actively, impacting both architects and the audiences that graphic artifacts are intended for. It builds on my own extensive background working on design competitions while at Behnisch Architekten in Stuttgart, Germany, where I became attuned to the discourse following upon the reception of drawings in what was and remains a highly celebrated public process of commissioning in a tremendously rich and progressive design culture. Lastly this project is motivated by both recognition of how far sustainability concerns have shifted construction and design thinking in a very short period of time and how examples of contemporary, ecologically 'relational' graphics indicate further transformations underway in the very conception of the task of architecture. ${ }^{1}$

\section{PICTORIAL NARRATIVES FOR SUSTAINABLE ARCHITECTURES}

For a proposal that registers prominently in the public consciousness, architectural design in the conceptual stages is a pictorial narrative of an arrangement of spaces and technical systems and a value laden declaration of what a place in the world might look like in the future and how it could fulfill larger cultural aspirations. The architect communicates an ethos of spatial 
and material relationships in complex graphic shorthand, with choice of image type and combinations of types serving as forms of conjecture with multiple ways of activating the imaginary. ${ }^{2}$ Graphic atmospheres and compositional arrangements are constructed to sway audiences of clients, juries and publics to assert shared commitment to a course of action that will lead to a significant reshaping of the spaces of the city.

Notable in contemporary green design culture is the preponderance of visionary architectural and urban design proposals that emphasize drawings where self-contained, 'biospheric' qualities of buildings are emphasized. In this genre of iconic sustainability, a crisply delineated figure, rivaling in universality the sculptural perspectival presence of the midcentury modernist tower, appears as the solution that absorbs the complexity of concerns that an environmentally progressive architect is expected to account for. The project as portraiture occupies the center of the picture field, where arcs of lines emanating from vanishing points coalesce. Flanking buildings reinforce the centrality of the subject, their supporting role made all the more apparent when cut off by the picture frame. If people occupy the image, they are few and scattered. Green walls and sky gardens adorn a taut shell.

Such graphic portrayals, unapologetically insular and of high and even-handed resolution, may ironically be a path to the realization of projects that contribute to greater levels of environmental fragmentation. Representing projects as isolated from one another and from the larger world short circuits opportunities to consider the synergistic performance of ensembles of buildings and landscapes, synergies that might significantly alleviate the burden - thermal, luminous, other - that works of architecture in their isolation are too frequently expected to shoulder. Such architectural imagery as narrowly circumscribed environmental problem formulation, a building island biogeography, operates in contrast to an emphasis on more open graphic propositions of ensembles and sets embedded in a larger system of relations. ${ }^{3}$

Scaffold-like combinations of images that marry dimensions of experience and performance, are, it is hypothesized, more inclusive in fashioning sustainable environments in that the designer communicates potential between drawings, between systems (structural systems, ecosystems, others) and between near and far horizons, and invites others to participate in the process of actualization. Lee Rust Brown advocates for such fullness of partiality: "The fugitive image or fragment speaks more compellingly to our interpretive superiority than the finished contours of a generic set piece." ${ }^{4}$ Proclaiming creative license while relinquishing full ownership over the process, such drawings have potential to instantiate discussion as to how a proposal represents an alteration and activation, intensification or dissipation of environmental conditions. The motivation underlying graphic production pertains less to assigning fixed entities to set spatial coordinates and more toward ensuring the primacy of desired relations as the design proceeds to higher levels of specificity and commitment.

\section{GRAPHIC CONSTELLATIONS, ABSENCES AND ATMOSPHERES}

In what follows, I will discuss a strategic sequence of graphic operations associated with critical environmental engagement and that build from the idea of works of architecture as materially and energy efficient and as contributors to ecologically functional cities. An aphoristic approach to the making of drawings, one that is suggestive and prompting versus fixed, involves complex layering, conscious absence and the embracing of tension and irony. A self-critical attitude toward the generation and ecology of imagery may lead to more transformative and environmentally responsive architectures.

\subsection{A Shimmering Through of Varying Density}

Considering the related and rapid trajectories of urban growth, mass species extinction, and diminished air and water quality, all occurring within a context of climate change, it is critical that designers consider with greater urgency the ways cities and the architectures that make them up can serve as loci of ecological possibility. By borrowing conceptual frameworks from the domain of landscape ecology and recognizing the city as a polycentric ecosystem, one of enormous environmental consequence and capable of directing, impeding and concentrating flows of energy, nutrients, water and light, designers are poised to assign a participatory function to the site and project in question (this role in addition and complementary to supporting life and activity within it). ${ }^{5}$ As the work of Rana Creek Living Architecture, Hyphae Design Laboratory and other innovative contemporary design firms bridging architecture and ecology provide evidence of, an architectural project may be variously identified as an oasis, feeder, redundancy, repository, decelerator (hydrological speed bump), safe passage, stopover, fold in the urban fabric or perhaps a channeling constraint that improves system-wide environmental performance. Even for a commission for a design intervention limited to one lot, by conceptualizing the projectto-be as contributor to a network, views of sustainability shift from a closed-loop internal system to the idea of architectures as systems embedded in those of larger magnitude. ${ }^{6}$

One dimension of ecologically motivated graphic inquiry pertains to the form and character of connective vegetative structures - trees, hedgerows, swales, green roofs and green facades - introduced as part of architectural development that might link to a larger green network in the role of stepping stones, stopovers, contiguous corridor extensions or fortifying side channels. Whether dense or filigree, solitary or among the multiple, the introduction of matrices of green corridors in the urban fabric, nested within existing or anticipating new ones, adds an important dimension to the notion of infill. A site plan or perspective sketch provides indication of the spatial status and identity of the proposed landscape structure. This structure might reinforce and complement the performance and presence of buildings (providing shading and cooling while acting as a 


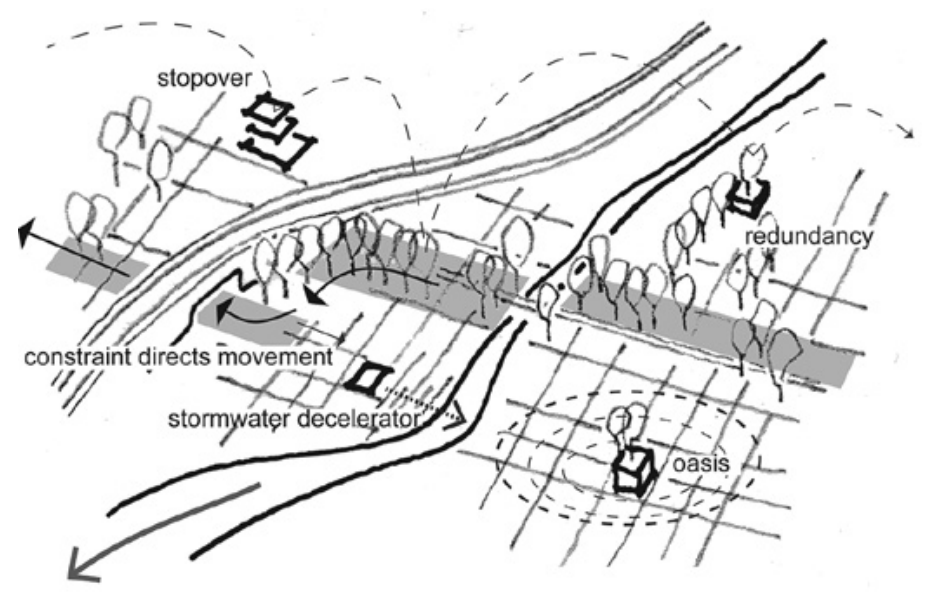

Figure 2.1.1: Potential contributions of individual sites to a polycentric urban ecological system

living billboard) while assuming its own pattern-like identity. A rendering might fittingly and abstractly denote both the sharp distinctiveness and the open and diaphanous qualities of the patterning of green, that is to say its loose precision.

A shift from green to blue instantiates consideration of how built forms and landforms cooperate in the creation of ecologically productive hydrological geometries. Three-dimensional studies describe a project as both within and as a watershed. Site sections describe the rhythmic patterning of architectural and landscape architectural elements that shape the flows of storm water, from clouds to buildings to urban waterways, and articulate 'steps' in processes of cleansing. Hybrid section perspectives of buildings and landscapes simultaneously depict spatial interaction at a strategic 'break' (plane of section cut) and a broader, persistent continuity (elements of significance such as water bodies and other mediums of conveyance extend the picture field in front of the cut). Water is harvested and passes into a project, is removed temporarily from the hydrological cycle, and put to work in a manner that leads to its treatment. Its release - to constructed wetlands and naturally occurring urban waterways - is timed to support ecological conditions, providing predictable flows and levels (hydroperiods) in an era of climatic uncertainty. While the Trevi Fountain celebrates the introduction of clean water to the city of Rome, a speculative contemporary design undertaking might involve the humble honoring of the successful completion of water's arduous journey through the architectures, infrastructures and open spaces of the city.

Certain places deserve greater graphic attention and intensity due to their location and/or intended use, and because their futures have taken on a heightened level of contestation and attention due to the commissioning of projects there. An actively attuned manner of representation is one in which graphic crystallization - moments of relatively high resolution and energy - bring out in

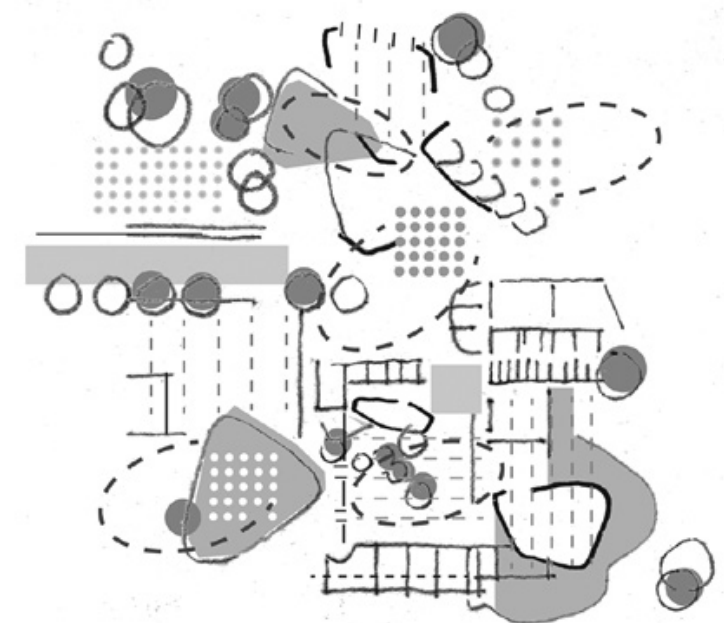

Figure 2.1.2: A shimmering through of varying density (interpretation of Behnisch Architects competition entry for Altana Pharma, Constance, Germany, 2002)

relief places of coalescence of ecological and human activity. The punctured incompleteness of aerial site collages and perspectives in Witherford Watson Mann Architects' competition winning "Bankside Urban Forest" neighborhood plan proposal (a project as "landscape stitching," or the knotting of the diffuse and concentrated) provides an instance of this approach. ${ }^{7}$ The design team renders underutilized and neglected intersections and streets in a haunting, simultaneously green and gritty collage-like manner. People can see the potential of these urban spaces; a conceptual and communicative path is created for the district to acquire renewed life.

Thinking of any project and site as but one locus within innumerable vanishing points, as the charged occasion of two or more systems coming into contact, differs from an island-like, selfcontained vision of a proposal purported as progressively green. Christian Kandzia of Behnisch Architekten describes architecture as "a shimmering through of varying density" (the graphics the firm produces clearly support this outlook). ${ }^{8}$ Spatial and material ensembles radiate different effects at a range of intensities and through variously charged atmospheres. Lines, colors and words that play with these illuminated significances stimulate a succession of impressions paralleling our encounters with the actual. In other words, more abstract representations in their suggestive particularity capture our experience more fittingly than those of a hyperrealist nature. Graphic constellations of varying brilliance alight on branching line works and enliven the compositional matrix. Such a capaciously unburdened view resonates with Cary Wolfe's thoughts on the sublime:

It is an incommensurability that carries ethical force, for it serves as a reminder that the heterogeneity of the world cannot be reduced to a unified rule or reason. And this incompleteness in turn necessitates a permanent openness of any discourse to its other. ${ }^{9}$ 


\subsection{Stills of Dynamically Interacting Parts}

In their recent work The West Without Water, Ingram and Malamud-Roam speak to a profound and problematic ecological estrangement:

\begin{abstract}
Water is uniquely vulnerable to overuse. It falls freely from the sky, giving a false sense of abundance. Water policy in the West has made it a resource that is easy to seize and exploit by those with the power and will to do so. As yet there is little incentive to conserve water for the common good, despite scores of ingenious water conservation proposals. Society in the West lacks any sense of urgency concerning the growing scarcity of this lifesustaining resource. People in the region are oddly estranged from their natural relationship - living in human-created oases of concrete, manicured lawns, and air-conditioned homes. Few are aware of the origins of the water flowing out of their taps, which is probably due, in part, to the complexity of the region's modern, highly engineered hydrology. ${ }^{10}$
\end{abstract}

How can green architectures that increasingly bracket and separate by virtue of tight shells with low surface-to-volume ratios, that are in so many ways an expressive elaboration of separateness (in contrast to the fashioning of connective tissue that is at the heart of landscape architecture), assist in overcoming this estrangement? What is the role of graphic conjecture in this process?

We have considered more seamless relationships between architectures and landscapes, where the performance of the former contributes to that of the latter. A contrasting approach, pressed into service in making architectures that facilitate ecological re-familiarization, may begin ironically through selective graphic de-emphasis. Exaggeration and strategic discrimination, for example removal of graphic content to place distant entities in sudden proximity, calls attention to codependencies not otherwise perceived (such a strategy contrasts with a more even-handed representational approach that may encourage physical realization of weakly connected parts). The graphic elimination of the middle ground, the building envelope or what Leatherbarrow would describe as the practical horizon, highlights potential linkages between events and ensembles immediately at hand (Leatherbarrow's equipmental horizon) and surrounding phenomena (the environmental horizon) that deserve attention and that perpetuate our quality of life. ${ }^{11}$ As Valerie Gonzalez says of the Islamic architecture of Andalusia, one "draws a topography by juxtaposition of various isolated elements." 12 Such is the case with Smoot Allen's diagram of technical performance in their Grand Egyptian Museum competition entry where the distant water source and the (ever) presence of water within the project are collapsed in one world. ${ }^{13}$ The project acquires meaning through expression of absence, transmittance across desolation, and the act of pulling

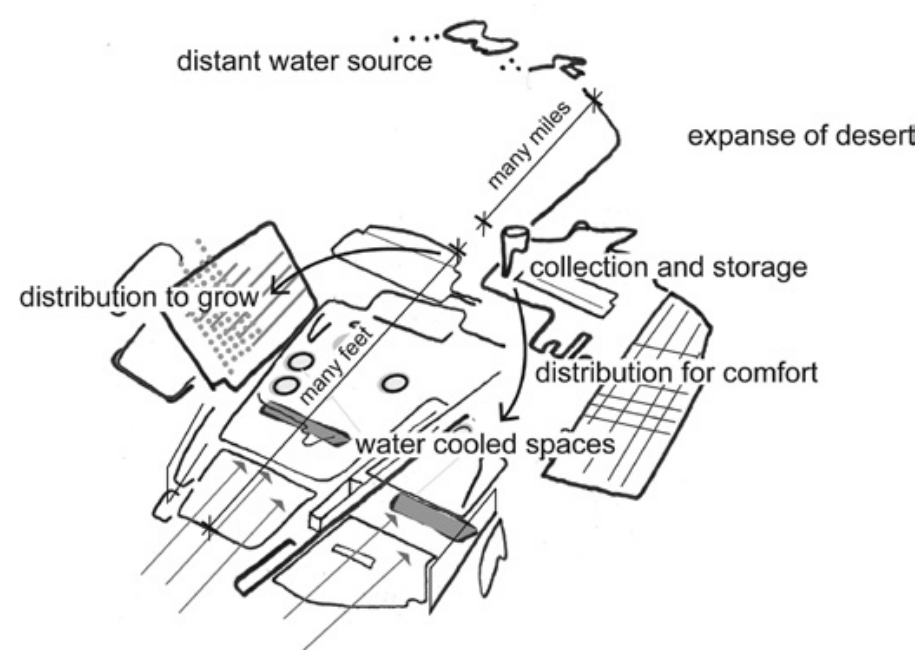

Figure 2.2.1: Diagram overlay of Smoot Allen's aerial perspective for their Grand Egyptian Museum competition entry

the figure of the 'building' apart to establish new speculative ground. Displacement provides the basis for new affinities and forms of recognition.

Following its initial erasure, the invisible practical horizon reappears in a new guise such that architectural function can proceed in a manner that will not result in significant environmental impact. If modernist architecture involves reliance on extractive technologies (mechanical thermal control systems permitting large fields of glass allowing direct connections to the landscape) that in the end compromise that which transparency enables unobstructed contact with, an appropriately open ecological architecture is one tied perceptibly to resource use. This tying may involve collapsing multiple functions (horizons) into fewer, thinner and more immediate assemblies. For instance, in the John and Frances Angelos Law Center at the University of Baltimore, Behnisch Architekten deploys minimized planes of 'smart' concrete to circulate air and absorb heat and that operate in concert with heat exchangers embedded in facades. ${ }^{14}$ The ensemble allows for the elimination of ductwork. Mechanical equipment is transformed into furnishings; learning communities occupy volumes once taken up by air distribution networks.

As the thermal envelope, heretofore the locus of expression in sustainable architecture, becomes smarter and minimized, the opportunity beckons for other elements and systems - water harvesting systems for example that provide thermal mass, seismic dampening, fire suppression and sound attenuation - to emerge in fuller figural and functional expression. Smoot Allen's aforementioned Grand Egyptian Museum competition entry offers an example of the kind of preparatory work necessary to achieve such architectures. The introduction of a ribbon of water to the graphic field of the project allows for the delineation of the museum itself as a water-cooled thermoregulatory device: a condenser, distributor, mister and micro-climatic phase changer. 
Arrays of ecological gear wash over point, line and plane.

As the Angelos Law Center illustrates, we are witnessing a reconstitution and hybridization of Leatherbarrow's three horizons, the environmental and equipmental (and the visual and analog) now standing alongside, enmeshed with and entering in and out of the practical. The environmental horizon becomes more immediate and present while larger scale 'prosthetic' equipmental sets are deployed in the urban landscape. Perception shifts from distant contemplation (landscape appreciation) to a finer grained, oscillating system of nature/cultures, as with the Hyphae Design Laboratory's water systems designs that operate as connectors and mediators amongst complexes of urban buildings and landscapes. ${ }^{15}$

Drawings such as those produced by Smoot Allen and Behnisch Architekten instantiate this process of architectural reorientation and deepened environmental perception; these graphic artifacts stimulate awareness of correspondences between architectural 'atmosphere' and systems processes, where scaffold-like forms are enlisted for the effects they produce. One can investigate these possibilities at play by depicting characteristics of performance and experience side-by-side or where one is overlaid atop the other, as in Snøhetta's drawings for the Powerhouse projects. ${ }^{16}$ And where one often sees performance attributes layered over experientially oriented drawings such as perspectives or section perspectives, it might be valuable to consider the opposite, where indications of experiential opportunity are superimposed on representations geared toward system performance. It may even be that symbols and registers intended to convey information in a drawing can eventually acquire the status of physical or virtual/ digital presences and serve as didactic tools in realized projects.

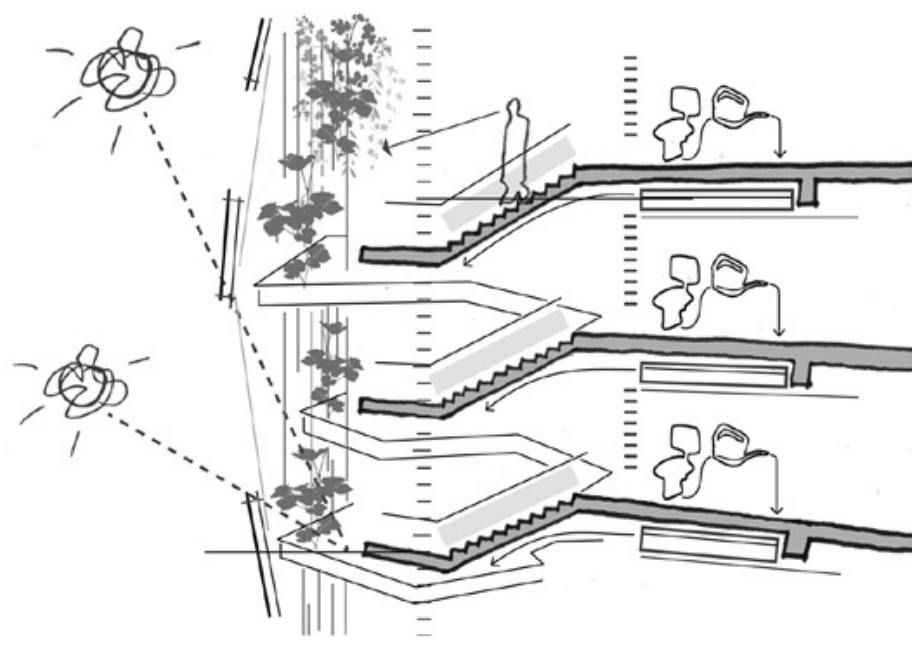

Figure 2.2.2: Experiential and performance-based dimensions of architecture described in one image (based on winter 2014 architectural studio project by Carolyn Lieberman, Samuel Ridge and Carolyn Tucker)
Within an overall board composition, rather than segregation of drawing types, precincts of interest areas are formed out of multiple, complementary types including the performance/ experience hybrids described above. Within these precincts and across types, one assigns distinct stylistic characters to sets of entities and phenomena with shared attributes (some sets are freehand, others hardline, others fashioned from collage cutouts) in order that the viewer draws correspondences between drawings. Several precincts combine to describe a project composed of and made compelling by a loose-knit synthesis of multiple narratives. The armature assembled is both encompassing and open.

\subsection{Flow Beyond}

Elements with sufficient fullness and freedom (however fleeting) extend beyond a site and break from the confines of the solitary picture plane. To sweep in lines in a manner commensurate with ecological significance is to reinforce the notion of the site as one spatio-temporal event within vast and shifting envelopes, pressures and influences. Elements and systems that might be represented in this way include rivers, associated wetland networks and other urban waterways, connective urban green space, climatic forces, subsurface flows, topography, and axes between notable landscape features. ${ }^{17}$ Some of these are visible and regular in occurrence, others more episodic in nature. Some of these are less strictly ecological but nevertheless play important roles in the environmental circumstances that the project in question contributes to.

A dash or stippling traces a delicately complex patterning of migration. An arc or encircling designates influences of higher ambient magnitude. An array of arrows renders visible the sway of an atmospheric field. A fill spills over its boundaries and saturates adjacent districts (the frame is introduced for the very purpose of being exceeded). A watery wash is the medium through which beings pass and around which people gather (what we draw and are drawn toward). Given interest in calling forward, pushing beyond and breaking free, graphic language in its tremendous versatility permits endless variation of meaning and emphasis through line thickness, character and style, and as such compares favorably with the constrained linear narrative sequence of the written word. One and the same graphic can delineate the physical while conjuring the ineffable.

Different sets of symbols proceed in and out of drawings along different trajectories and toward distinct ends, on to the limitless horizon (edge of a sheet). Flows break down the insularity of any one drawing, redistribute elements across the page, and highlight and connect kindred elements in different drawings of dissimilar scale. This overarching presentation/board composition strategy further reinforces the notion of a project's fundamental incompleteness, ongoing conceptual construction, and inevitable condition as both center and edge. 


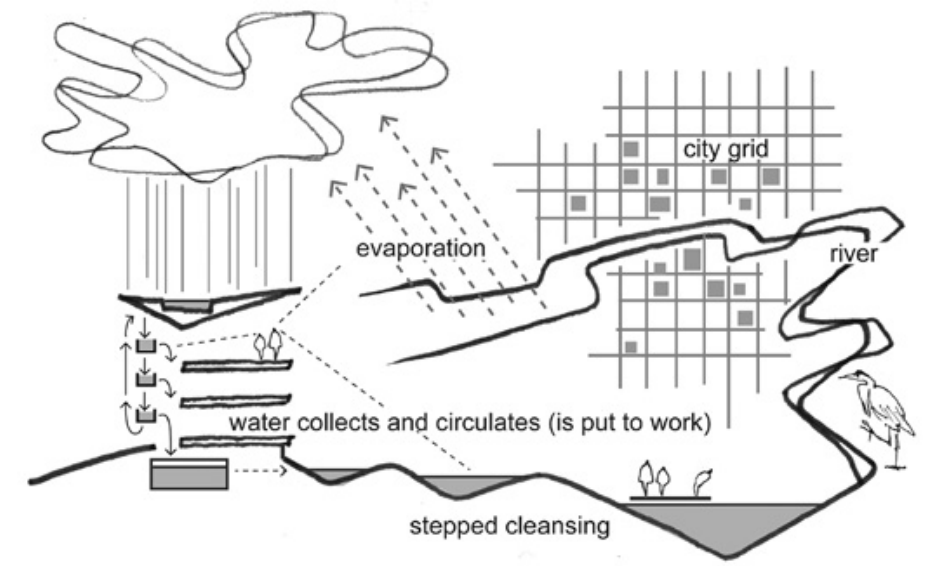

Figure 2.3.1: Ecological flows as graphic linkages

\section{RESEARCHING GRAPHICS FOR THEIR ENVIRONMENTAL EFFECTS}

Pragmatist philosopher Mark Johnson argues: "The very meaning of ecological and sustainable design is an open-ended imaginative experiment." ${ }^{18}$ The question of what constitutes a sustainable approach requires ongoing speculation as much as adherence to and playing out of variations on a grounded set of conventions. Such speculation includes graphic inquiries that prefigure subsequent acts of architectural production (as the late German architect Guenter Behnisch told his design team twenty-four hours before a competition deadline: "Remember, you are not designing a building. You are designing drawings that describe aspects of what a building can be"). ${ }^{19}$ As with any language, architectural graphics involve a set of choices intended to produce effects. While there are numerous possible effects of imagination and environmental discernment that architects might simulate through the forms of graphic communication discussed in this essay, I will concentrate on three and enumerate research questions and opportunities associated with each:

The effect of heightened awareness of the ecologically relational Evocative depictions of contextual influences bring a more outward disposition to sustainable architecture, a view that it must engage issues other than and in addition to the internal dynamics of building performance. Intensified through exaggeration and relief, 'ecological' graphics can stimulate discourse about ways individual projects can participate in and contribute to broader ecosystem function.

In drawings identified as motivated by a goal of promoting enriched dialog pertaining to relationships between site-scale interventions and larger ecological context, what specific types of relations are depicted? How do drawings highlighting building/ ecological relationships influence dialog in subsequent design phases, both within the design team and between the team and clients? How in turn does the formation of an ecologically enriched graphic and verbal narrative influence project outcomes?
The effect of conceptual reorientation through graphic omission Omission of content and sudden juxtaposition of elements and conditions that at first appear disparate enable recognition of desirable and necessary environmental dependencies. Drawings can collapse scales and depict continuities between the immediate and proximal and the distant and vast.

How might selective omission of content enable people to see correspondences between systems and entities, buildings and surroundings that they might not otherwise recognize? Might these omissions foster conceptual reconnections that influence dialog amongst the design team and between the team and the client in a manner that leads to more ecologically responsive architectures?

The effect of invitation through conscious graphic ambiguity In the conceptual phases of design the architect stakes out a set of possibilities for intervening in the world by engaging a process that marries the highly specific with graphic elements and combinations more open in nature. This mixture of precision and indeterminacy is of a consciously suggestive, distinctive kind, productively constraining the meanings to be inferred, acknowledging the gap that exists between artifact and execution, and making evident the fiction that has much to do with a drawing's potential as productive instigator. ${ }^{20}$

To what extent does an initial graphic approach characterized by conscious ambiguity encourage audiences to offer interpretations that may add new dimensions to the design problem under scrutiny? Are architectural graphics of this nature an insider's language, comprehensible only to those fluent in design and visual thinking, or does such an approach hold more universal appeal? To what extent do 'invitational' graphics engender what sociologist Niklas Luhmann describes as 'second order observation,' where attention is directed toward the interpretations of others as much as the artifact itself? ${ }^{21}$ How does this influence design processes and outcomes? How might these second order observations suspend the process at key moments in order to, as Guenter Behnisch encourages, "give a chance to the things that fail to get the attention they deserve, but still may mean more to us than those that in any case force themselves upon us"? 22

These three possible effects point to a larger question pertaining to the ability of architectural graphics to galvanize increased environmental concern by rewarding the intelligence of viewers and by ensuring the space of possibility for alternative views and altered courses. In this outlook, graphic production is ongoing questioning of intentions and desired influences, where the skill of the executor corresponds to belief in a public's environmental values and interests and willingness to be challenged and moved. To quote Lee Rust Brown:

The common world is realized not by actual 
obedience to a doctrine but by the recognition of a common place, the visionary place where manifold individual paths of departure can stand in mutual criticism and mutual respect. ${ }^{23}$

The designer first authors and thereby facilitates paths of departure. Meaning resides not in the drawing but in its capacity to leap off the page, offer arresting cues, suggest a unity that is continually deferred, and invite ongoing negotiation, self-critical awareness and discovery in a social and ecological situation.

\section{ENDNOTES}

1. See Bourriaud 2002 for a treatment of Relational Aesthetics.

2. Look no further than the design proposals of the finalists for the 9/11 World Trade Center Memorial Competition for skillfull manipulation of images ensuring prominence of that which is significant, in this case a place for mourning, memorialization and the silent expression of the voiceless in the context of a development project in which $90 \%$ of the program is given over to private sector/spec office use.

3. Speculative Ecodistricts-based development projects furnish examples of a syngeristic inter-building graphic approach; see: http://ecodistricts.org/

4. Brown 1997, 111.

5. Harvard University Landscape Architecture professor Carl Steinetz argues that decisions made at the lot scale are inconsequential without effective, environmentally oriented planning policies at the region scale. While I would not disagree with the importance of getting the bigger picture right, given the kinds of projects I have dedicated my efforts towards, I have to proceed believing in the catalytic potential of the solitary individual (with an ecological contribution in excess of its size) and the benefits of building up an aggregate of connective projects.

6. For a thorough explanation of a hierarchical theory of ecosystems, see: O’Neill et al. 1986.

7. See: http://www.wwmarchitects.co.uk/subpage/public_ spaces/bankside_urban.php?view=ps

8. The quote comes from Christian Kandzia's lecture at the University of Oregon on October 21, 2011. Along similar line of thought, Valerie Gonzalez describes architecture as a "prism of artistic wonderment"; see 2001, 53.

9. Wolfe 2010, 218.

10. Ingram and Malamud-Roam 2013, 212.

11. See Leatherbarrow, 2000.

12. Gonzalez 2001, 109.

13. See Smoot Allen 2007.

14. See http://behnisch.com/projects/521

15. See: http://hyphae.net/journal/

16. See: http://powerhouse.no/en/snohetta-eng/

17. As for the latter, see Moorish 1996.

18. Johnson's quote appears on the back cover of my book Ecology and the Architectural Imagination 2014.

19. Personal correspondence, July 1994.
20. For a consideration of the political motivations of aesthetic inquiry - and the space of possibility of alternative views that certain aesthetic domains can open up - see Eagleton 1990.

21. See Luhmann 2000.

22. From transcript of lecture that Guenther Behnisch delivered at the University of Oregon School of Architecture and Allied Arts, May 1990.

23. Brown 1997, 24.

\section{REFERENCES}

Bourriaud, Nicholas. 2002. Relational Aesthetics. Translated by Simon Pleasance and Fronza Woods with the participation of Mathieu Copeland. Dijon: Les presses du reel. 1998.

Brown, Lee Rust. The Emerson Museum: Practical Romanticism and the Pursuit of the Whole. Cambridge, MA: Harvard University Press. 1997.

Eagleton, Terry. The Ideology of the Aesthetic. Oxford, UK: Basil Blackwell. 1990.

Gonzalez, Valerie. Beauty and Islam: Aesthetics in Islamic Art and Architecture. London: I.B. Tauris Publishers. 2001.

Ingram, B. Lynn, and Malamud-Roam, Frances. The West Without Water: What Past Floods, Droughts, and Other Climatic Clues Tell Us About Tomorrow. Berkeley, CA: University of California Press. 2013.

Leatherbarrow, David. Uncommon Ground: Architecture, Technology, Topography. Cambridge, MA: The MIT Press. 2000.

Luhmann, Niklas. Art as a Social System. Translated by Eva M. Knodt. Stanford, CA: Stanford University Press. 2000 (original German edition 1995).

Moorish, William Rees. Civilizing Terrains: Mountains, Mounds and Mesas. San Francisco: William Stout Publishers. 1996.

Muller, Brook. Ecology and the Architectural Imagination. New York: Routledge. 2014.

O'Neill, R.V., DeAngelis, D.L., Waide, J.B., and Allen, T.F.H. A Hierarchical Concept of Ecosystems (Monographs in Population Biology \#23). Princeton, NJ: Princeton University Press. 1986.

Smoot Allen. Augmented Landscapes (Pamphlet Architecture 28). New York: Princeton University Press. 2007.

Wolfe, Cary. What is Posthumanism? Minneapolis: University of Minnesota Press. 2010. 